\title{
Transition from eruptive to confined flares in the same active region ${ }^{\star}$
}

\author{
F. P. Zuccarello ${ }^{1,2}$, R. Chandra ${ }^{3}$, B. Schmieder ${ }^{2}$, G. Aulanier ${ }^{2}$, and R. Joshi ${ }^{3}$ \\ ${ }^{1}$ Centre for mathematical Plasma Astrophysics, Department of Mathematics, KU Leuven, Celestijnenlaan 200B, 3001 Leuven, \\ Belgium \\ e-mail: francesco.zuccarello@wis.kuleuven.be \\ 2 LESIA, Observatoire de Paris, PSL Research University, CNRS, Sorbonne Universités, UPMC Univ. Paris 06, Univ. Paris-Diderot, \\ Sorbonne Paris Cité, 5 place Jules Janssen, 92195 Meudon, France \\ 3 Department of Physics, DSB Campus, Kumaun University, 263001 Nainital, India
}

Received 3 October 2016 / Accepted 3 February 2017

\begin{abstract}
Context. Solar flares are sudden and violent releases of magnetic energy in the solar atmosphere that can be divided into two classes: eruptive flares, where plasma is ejected from the solar atmosphere resulting in a coronal mass ejection (CME), and confined flares, where no CME is associated with the flare.

Aims. We present a case study showing the evolution of key topological structures, such as spines and fans, which may determine the eruptive versus non-eruptive behavior of the series of eruptive flares followed by confined flares, which all originate from the same site.

Methods. To study the connectivity of the different flux domains and their evolution, we compute a potential magnetic field model of the active region. Quasi-separatrix layers are retrieved from the magnetic field extrapolation.

Results. The change in behavior of the flares from one day to the next - from eruptive to confined - can be attributed to the change in orientation of the magnetic field below the fan with respect to the orientation of the overlaying spine rather than an overall change in the stability of the large-scale field.

Conclusions. Flares tend to be more confined when the field that supports the filament and the overlying field gradually becomes less anti-parallel as a direct result of changes in the photospheric flux distribution, being themselves driven by continuous shearing motions of the different magnetic flux concentrations.
\end{abstract}

Key words. Sun: filaments, prominences - Sun: flares - Sun: magnetic fields - Sun: activity

\section{Introduction}

Solar flares are among the most energetic phenomena that occur in the solar atmosphere, and they can be divided into eruptive flares, which are associated with coronal mass ejections, and confined flares (see reviews of Schmieder et al. 2015; Janvier et al. 2015). These latter are normally not associated with a CME, and either no filament is present at all (Schmieder et al. 1997; Dalmasse et al. 2015) or the filament fails to erupt (Török \& Kliem 2005; Guo et al. 2010a). In addition to full and failed eruptions, there are cases where only a part of the filament erupts; such events are defined as partial erupting events. Partial eruptions may or may not be associated with a CME (Gibson \& Fan 2006; Liu et al. 2008, 2012; Tripathi et al. 2013; Kliem et al. 2014b; Zhu \& Alexander 2014).

The most energetic flares are commonly eruptive (Yashiro et al. 2005), even though confined, non-eruptive X-class flares have been reported (Thalmann et al. 2015; Sun et al. 2015; Harra et al. 2016) as well as CMEs with associated only C-class flares (Romano et al. 2014; Chandra et al. 2016).

The CSHKP model (Carmichael 1964; Sturrock 1966; Hirayama 1974; Kopp \& Pneuman 1976) and its extension in three dimensions (Aulanier et al. 2012; Janvier et al. 2013, 2015) can explain several observational signatures of the full (or

\footnotetext{
$\star$ Movies associated to Figs. 2, 3, and 5 are available at http://www . aanda.org
}

failed) eruptive flares, such as the presence of X-ray sigmoids, flare ribbons, and brightening motions along the ribbons themselves. In particular, Savcheva et al. $(2015,2016)$ have shown that the flare ribbons often coincide with the photospheric signature of quasi-separatrix layers (QSLs, Démoulin et al. 1996), i.e., thin layers characterized by a sharp gradient in the connectivity of the magnetic field. The brightening motions along the ribbons have been interpreted as the signatures of the slipping reconnection of the magnetic field lines through the QSL (Aulanier et al. 2006; Janvier et al. 2013; Dudík et al. 2014, 2016).

The morphology and evolution of flare ribbons can also give information on the overall topology of the system. Masson et al. (2009) have shown that circular flare ribbons are associated with the presence of a null-point topology in the corona, while parallel ribbons moving away from each other have been interpreted as an indication of quasi-separator reconnection occurring higher in the corona (Aulanier et al. 2012).

From a theoretical point of view, a key feature of the standard model for solar flares is the presence of an outward moving magnetic flux rope, i.e., a topological structure made up of twisted magnetic field lines that wrap around an axial magnetic field line. Structures compatible with magnetic flux ropes have been observed on an active region scale (Canou \& Amari 2010; Jing et al. 2010; Guo et al. 2010b; Green et al. 2011; Savcheva et al. 2012; Gibb et al. 2014; Jiang et al. 2014) and on a larger scale in coronal 
cavities (Gibson et al. 2010; Rachmeler et al. 2013; Gibson 2015; Bak-Steslicka et al. 2016). The standard flare model requires a mechanism that triggers the onset of the flux rope eruption, resulting in the phenomenology observed during solar flares.

Different triggering mechanisms have been proposed and discussed in the literature (see Forbes 2010; Chen 2011; Aulanier 2014; Filippov et al. 2015; Schmieder et al. 2015, for a review), but essentially the equilibrium of a magnetic flux rope embedded in an overlying magnetic field is determined by two competing effects: the outward-directed magnetic pressure between the flux rope and photosphere, and the inward-directed magnetic tension of the overlying field.

In the torus instability or catastrophic loss of equilibrium model (Forbes \& Isenberg 1991; Kliem \& Török 2006; Démoulin \& Aulanier 2010; Kliem et al. 2014a) it is the onset of an ideal magneto-hydrodynamic instability that leads to the disruption of this equilibrium, while in the breakout model (Antiochos et al. 1999; Lynch et al. 2008; Zuccarello et al. 2008, 2009; Karpen et al. 2012) it is the onset of a resistive instability.

Assuming an overlying external field $B_{\mathrm{ex}}$ that scales with the height $z$ from the photosphere as $B_{\mathrm{ex}} \propto z^{-n}$, in the torus instability model the system becomes unstable when the apex of the axis of the magnetic flux rope reaches a critical height $z_{\text {cr }}$ where the decay index $n$ of the external overlying field $B_{\text {ex }}$ becomes larger than a critical value $n_{\mathrm{cr}}$. The results of several MHD simulations place $n_{\text {cr }}$ in the range [1.3-1.75] (Török \& Kliem 2005, 2007; Fan \& Gibson 2007; Isenberg \& Forbes 2007; Aulanier et al. 2010; Kliem et al. 2013; Amari et al. 2014; Inoue et al. 2015; Zuccarello et al. 2015, 2016). Attempts to estimate the decay index at the onset of solar eruptions have also been made using both limb observations and stereoscopic observations (Filippov \& Den 2001; Guo et al. 2010a; Filippov 2013; Zuccarello et al. 2014; McCauley et al. 2015). These studies have found an "observed" critical decay index $n_{\mathrm{cr}}^{\mathrm{ob}}$ in the range [1-1.1], with this discrepancy between models and observations partially due to the different location where the decay index is computed (Zuccarello et al. 2016).

Contrary to the torus instability model, which does not require any particular magnetic field topology, the breakout model requires a multi-flux distribution. The eruption begins when a resistive instability sets in at the so-called breakout current sheet that exists between the arcade that confines the flux rope and the overlying field (Karpen et al. 2012). This reconnection removes the confining flux by transferring it to the neighboring flux domains. As a result, the magnetic tension of the confining field decreases and results in an eruption. For the breakout model to work two conditions must be satisfied: a null-point or quasi-separator must be present in the corona, and the flux of the confining arcade must be larger than the flux of the overlying field. Owing to the nature of the problem, i.e., evidence of reconnection occurring higher up in the corona, observational studies that clearly support the breakout model are quite rare (Aulanier et al. 2000; Mandrini et al. 2006; Chandra et al. 2009; Chen et al. 2016).

Both models address the triggering of filament eruption, but what determines whether a filament eruption results in a CME or in a failed eruption? Many questions have to be answered: does the trigger mechanism affect the eruptive/failed behavior of the flare, and if so how? How important is the magnetic environment of the active region?

In order to address these questions and to understand what causes confined or eruptive flares, we study a series of flares that occurred between 2014 April 15 and 16 in active region
Table 1. Details of compact and eruptive flares.

\begin{tabular}{|c|c|c|c|}
\hline \multicolumn{4}{|c|}{15 April 2014} \\
\hline $\begin{array}{c}\text { Flare } \\
\text { Number }\end{array}$ & $\begin{array}{c}\text { Flare onset } \\
\text { (UT) }\end{array}$ & $\begin{array}{c}\text { Flare class } \\
\text { GOES }\end{array}$ & $\begin{array}{c}\mathrm{CME} \\
\text { association }\end{array}$ \\
\hline 1 & $05: 56$ & - & No \\
\hline 2 & $06: 15$ & - & No \\
\hline 3 & $06: 59$ & - & No \\
\hline 4 & 09:15 & C8.6 & $10: 24$ \\
\hline 5 & $12: 34$ & C3.6 & $14: 00$ \\
\hline 6 & $14: 37$ & - & No \\
\hline 7 & $16: 56$ & - & No \\
\hline 8 & $17: 53$ & C7.3 & $18: 48$ \\
\hline 9 & $19: 22$ & - & No \\
\hline 10 & $20: 55$ & - & No \\
\hline 11 & $21: 39$ & - & No \\
\hline 12 & $22: 48$ & - & No \\
\hline 13 & $23: 40$ & - & No \\
\hline \multicolumn{4}{|c|}{16 April 2014} \\
\hline 14 & $01: 10$ & C1.9 & No \\
\hline 15 & $02: 42$ & - & No \\
\hline 16 & $03: 20$ & - & No \\
\hline 17 & $03: 48$ & - & No \\
\hline 18 & 05:02 & - & No \\
\hline 19 & $06: 37$ & C1.8 & $X$ \\
\hline 20 & $07: 14$ & - & No \\
\hline 21 & $08: 36$ & C5.2 & No \\
\hline 22 & $09: 20$ & - & No \\
\hline 23 & $10: 42$ & - & No \\
\hline$\overline{24}$ & $\overline{12: 42}$ & C7.5 & No \\
\hline 25 & $17: 30$ & $\mathrm{C} 2.0$ & No \\
\hline 26 & $19: 54$ & M1.0 & $X$ \\
\hline
\end{tabular}

Notes. Flares indicated in boldface are shown in Fig. 2, while the flare observed in $\mathrm{H} \alpha$ and also presented in Fig. 4 is underlined. The "-" indicates small flares that are not reported by GOES, " $X$ " indicates CMEs visible in LASCO, but not associated with the filament activity/eruption.

NOAA 12035 and that resulted in full eruptions on April 15 and in failed eruptions on April 16. The paper is organized as follows. In Sect. 2 we present our observational datasets. The analysis of the magnetic topology of the active region is presented in Sect. 3. Finally, in Sect. 4 we discuss our results and conclude in Sect. 5.

\section{Observations}

The active region NOAA 12035 appeared at the east limb on 2014 April 11 with a $\beta$ magnetic configuration and crossed the west limb on 2014 April 23. During its disk passage it produced many small to medium class solar flares. The active region turned into a $\beta \gamma$ magnetic configuration on 2014 April 13. During the disk passage on 2014 April 15 and 16, the active region (located at S15, E20 to E08) produced eruptive and compact flares, respectively. The description of these confined and eruptive flares is given in Table 1.

The flares on April 15 and 16 were observed by SDO with a high spatial and temporal resolution. In the current study, we used data acquired by the Atmospheric Imaging Assembly (AIA, Lemen et al. 2012) on board the Solar Dynamic Observatory (SDO, Pesnell et al. 2012). AIA observes the full Sun with different filters in EUV and UV spectral lines with a cadence of 


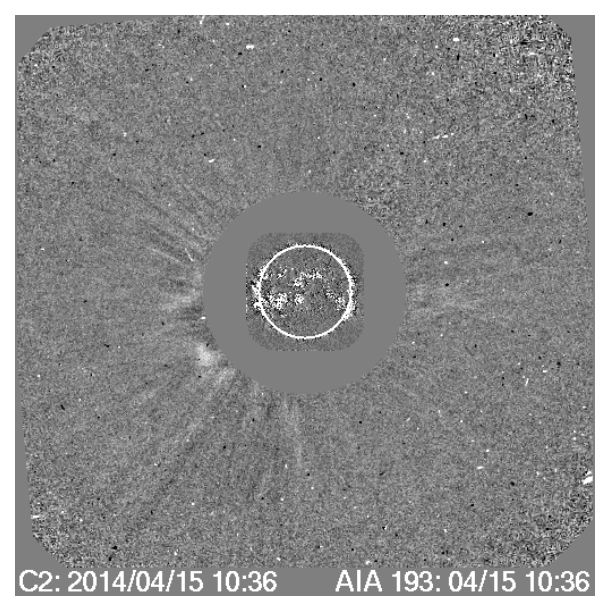

(a)

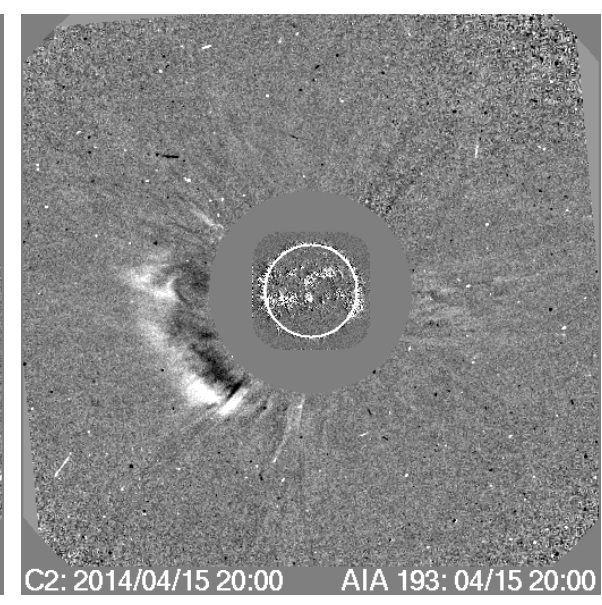

(b)

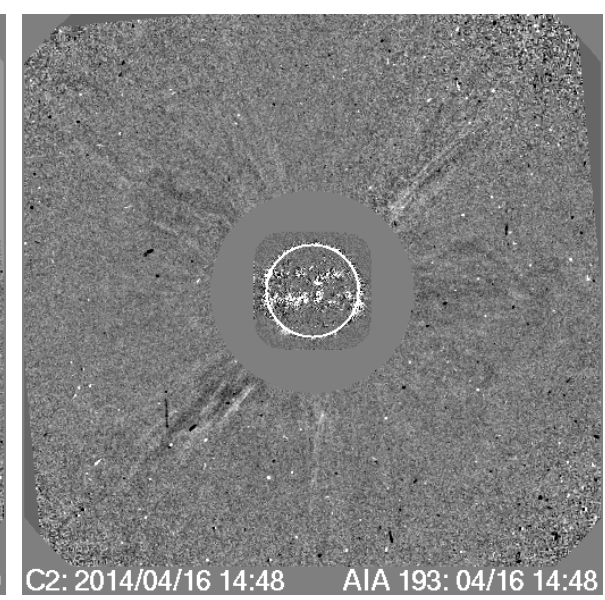

(c)

Fig. 1. SOHO/LASCO running difference images showing the associated CMEs for the eruptive flares. The last panel shows that no CME could be detected for the 2014 April 16 failed eruption.

12 s and a pixel size of 0 '”6. For this study, we used AIA $171 \AA$ and $304 \AA$ data.

For the chromospheric observations of one event on 2014 April 16, we used the $15 \mathrm{~cm}$ Coudé telescope equipped with a $\mathrm{H} \alpha$ filter from Aryabhatta Research Institute of Observational Sciences (ARIES), Nainital, India. These images are acquired with a cadence of $10 \mathrm{~s}$ and have a pixel size of 1 arcsec.

To study the magnetic topology of the active region, we used the data acquired by the Helioseismic and Magnetic Imager (HMI, Schou et al. 2012) aboard SDO. HMI measures the photospheric magnetic field of the Sun with a cadence of $45 \mathrm{~s}$ and a pixel size of 0 '.5.

Finally, to search for the possible CME associated with the flares, we used the LASCO C2 coronagraph data aboard the SOHO mission (Brueckner et al. 1995).

\subsection{LASCO/CME observations}

All the eruptive flares occurred on April 15. The CMEs associated with two of these eruptive flares are presented in Figs. 1a and $\mathrm{b}$. The CME associated with the C8.6 X-ray flare at 09:15 UT is first seen in the LASCO $\mathrm{C} 2$ coronagraph at 10:36 UT (Fig. 1a), and is characterized by a narrow angular width of 27 degrees and an average speed of $274 \mathrm{~km} \mathrm{~s}^{-1}$. The CME associated with the C7.3 X-ray flare that occurred at 17:53 UT is visible in the LASCO C2 field of view (FOV) at 20:00 UT (Fig. 1b), and has an angular width of 179 degrees and an average speed of $360 \mathrm{~km} \mathrm{~s}^{-1}$.

On April 16 no CMEs associated with the flares in the active region are observed. One example of the corona observed two hours after the flare that occurred at 12:42 UT is presented in Fig. 1c to show that no CME is detectable. However, we note that two CMEs are recorded on April 16 (see Table 1). After a detailed inspection of the LASCO movies we identified a poor CME around 06:30 UT that is too early to correspond to the flare at 06:37 UT, and a narrow CME directly towards the south at 20:00 UT that is again too early to correspond to the M1.0 flare. These CMEs could correspond to jet activity that characterize the eastern part of the active region.

\subsection{SDO/AIA observations}

All the flares of the AR 12035 considered in this study and listed in Table 1 were well observed by the AIA instrument on board SDO. Apart from the last one (flare 26 in Table 1), they are all low energy events, and correspond, for the strongest ones, to Cclass flares. During 2014 April 15 and 16, the region of interest produced three eruptive flares. Two of the eruptive flares, productive of CMEs, that occurred on April 15 are shown in the first two rows of Fig. 2 (in the AIA $304 \AA$ pass band).

Figure 2 presents the environment of the region around the time of the flare, and the black box in panel $b$ is focused on the AR 12035. A zoom of its evolution is presented in Fig. 3. As an example, the image at 09:15 UT shows a dark north-south oriented filament that was activated a few minutes before, and consequently bright arcades are observed around it. The flare emission reaches its maximum at 09:23 UT, while at 09:37 UT a round shape brightening can also be observed (Fig. 2b and accompanying Movie 1). Finally, between 09:40 UT and 10:03 UT dark stripes are seen to cross the active region from west to east (see Fig. 2c and accompanying Movie 1).

The second flare that we consider here occurred at 17:53 UT, when we see the activation of the filament that started to be aligned more east-west and with a second half-circle shaped filament at the north of it (Fig. 2e). Movie 1 shows a first failed eruption of the southern threads of the filament; afterwards the main body of the filament then starts to erupt at 17:51 UT, when circular bright arcades on the west of the filament are also visible (Fig. 2e and Movie 1). During the eruption the filament interacted with the environment and, similarly to the other eruptive flares, resulted in the ejection of plasma, visible as dark stripes around 18:33 UT (Fig. 2e and Movie 1). These dark, filamentary eruptive structures, which can be clearly seen in Figs. 2c and $\mathrm{f}$, had a duration of about $45 \mathrm{~min}$, and eventually produced the CMEs.

During the two days of observation the filament(s) evolved from being two separated filaments on April 15 - one relatively straight and oriented north-south at 09:15 UT and an upside down $U$ at the north of the first one clearly visible at 17:00 UT - to a complete east-west oriented sigmoidal filament on April 16 at 05:40 UT (see Fig. 3 and accompanying Movie 2). All failed eruptions observed on April 16 were initiated by an 


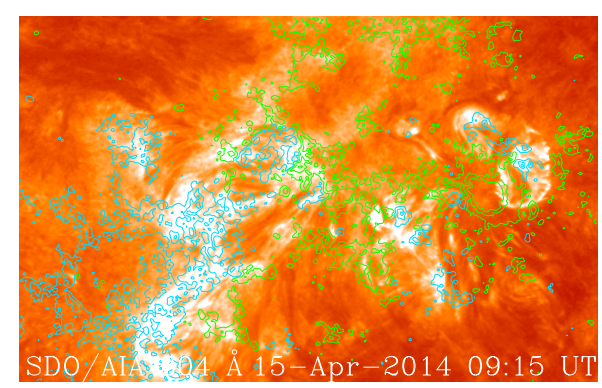

(a)

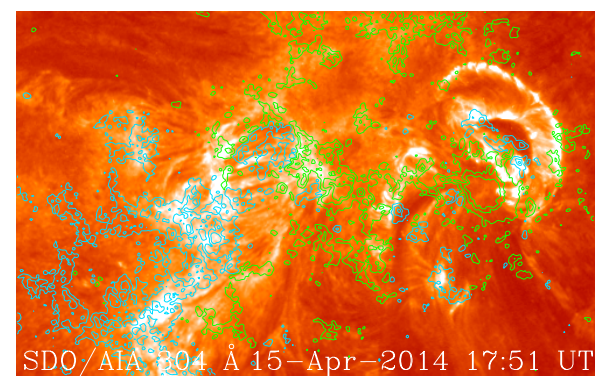

(d)

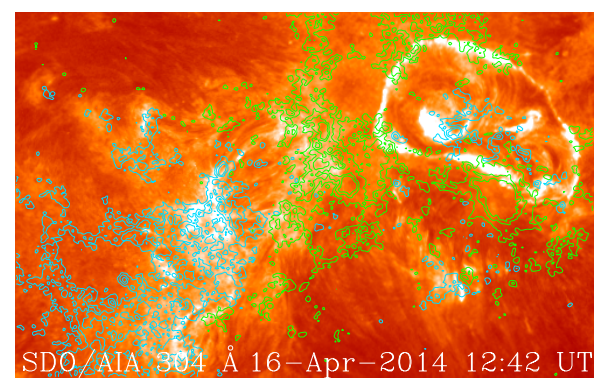

(g)

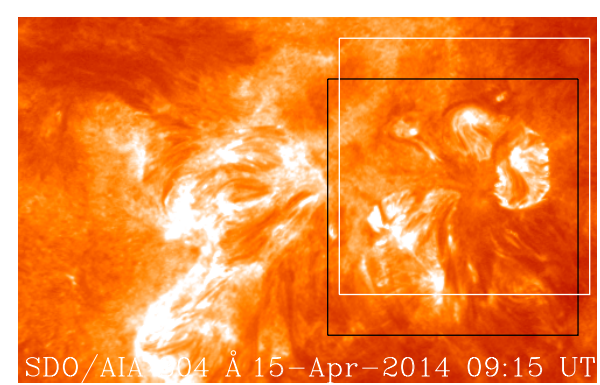

(b)

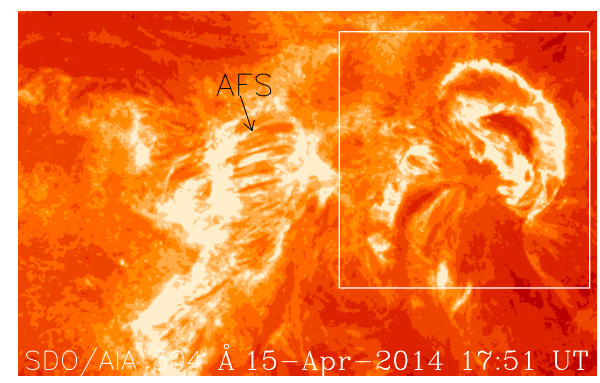

(e)

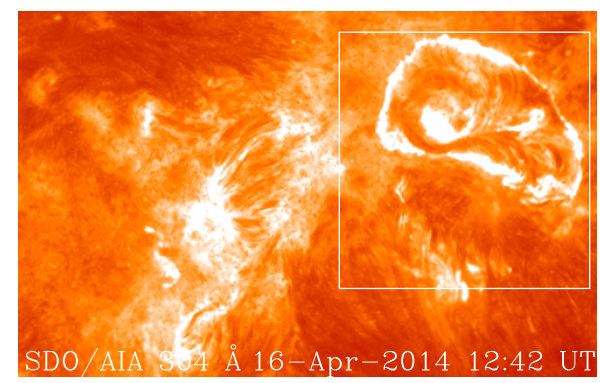

(h)

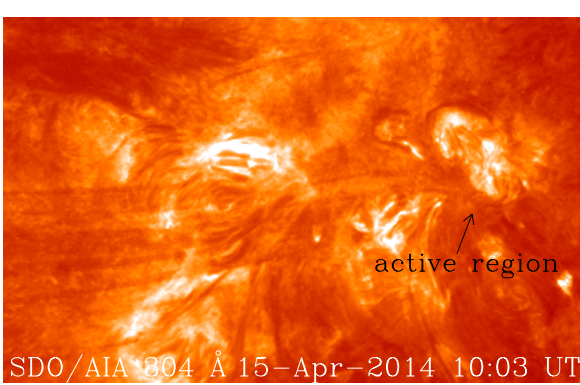

(c)

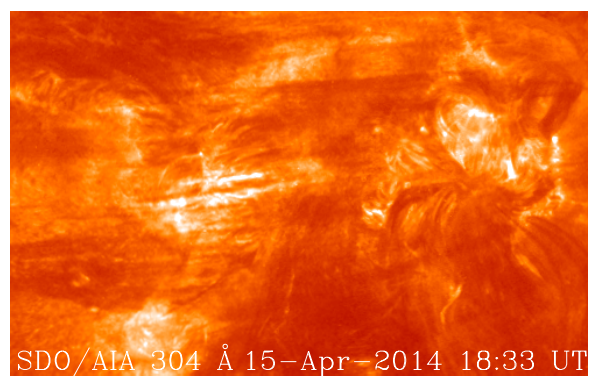

(f)

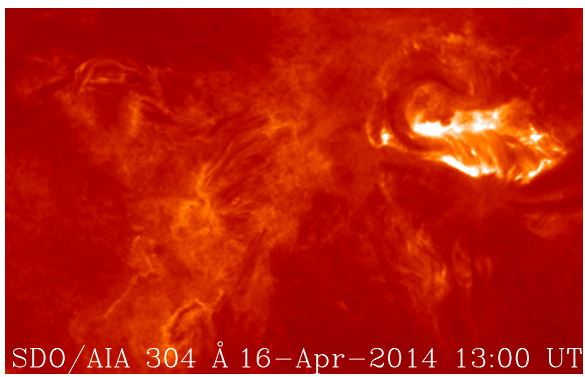

(i)

Fig. 2. Two examples of the C-class eruptive flares in the AR 12035 on 2014 April 15 in AIA $304 \AA$ (two top rows) and one example of confined flare on 2014 April 16 (bottom row). The FOV is [300 $\times 200]$ arcsec and the active region corresponds to the bright region on the right side of the images indicated by an arrow in panel c). The arch filament system (AFS) discussed in Sect. 2.4 is indicated by an arrow in panel e). The left column images are overlaid by HMI LOS magnetic field contours. Green/cyan contours (levels: $\pm 100, \pm 500, \pm 900$ ) represent positive/negative polarity, respectively. The black square in panel b) indicates the approximate FOV of Fig. 3, while the white square indicates the approximate FOV of Fig. 7. The temporal evolution is shown in Movie 1 (available online).

asymmetric failed eruption of the southwestern part of the sigmoidal filament.

One example of a compact flare that occurred on April 16 is shown in the bottom row of Fig. 2. Around the time of the onset of the flare, i.e., at 12:42 UT, we observe an oval shape of brightening around the AR 12035 inside the dark sigmoid and many filamentary structures in its southwestern end (Fig. $2 \mathrm{~h}$ and also Fig. 3). Until this time the dynamics is similar to what was observed the day before. However, at 13:00 UT a bright overlying arcade is seen over the AR 12035 and the dark material inside stops rising (Fig. 2i and Movie 2). The eruption concerned only the southern part of the sigmoid and was not able to drive all of the sigmoid to erupt. The two other failed eruptions, at 10:42 UT and at 20:00 UT, followed the same scenario. These three events lasted $15 \mathrm{~min}$ each. The eruption of 10:42 UT is well observed in $\mathrm{H} \alpha$ and is discussed in detail in the next section.

As a final remark, we note that recurrent jet activity is recorded at the southeast of the flaring activity. The study of this jet activity is beyond the scope of the present paper, which focuses on the transition from eruptive to confined flares in AR 12035.

\subsection{Ho observations}

In this section we discuss the failed eruption that occurred on April 16 at 10:42 UT and that is well observed in $\mathrm{H} \alpha$ from ARIES, Nainital.

The $\mathrm{H} \alpha$ image taken at 10:34 UT on April 16 (Fig. 4), shows the S-shaped filament (S) in the north of the active region, which was formed between April 15 and April 16 (see Fig. 3). Around 10:38 UT, the filament started to be activated, and at around 10:46 UT it broke in its center. The northern part of the filament remained in its original condition, while the broken part of it consisted of many threads (T) that are visible in $\mathrm{H} \alpha$ at 10:48 UT (Fig. 4) when the filament started to erupt in the west direction. However, the broken filament's southern foot point remained fixed. Eventually, the erupted part of the filament fell back on the solar surface, resulting in a failed eruption. Together with the filament eruption close to the breaking location of the filament, we observe the maximum flare brightening at 10:51 UT. Later on, we observe two flare ribbons (R1, R2) at 10:53 UT. Finally, we note that the dark $\mathrm{H} \alpha$ structure with a fan-like shape $(\mathrm{F})$ did not expand after 10:58 UT (see Fig. 4). 

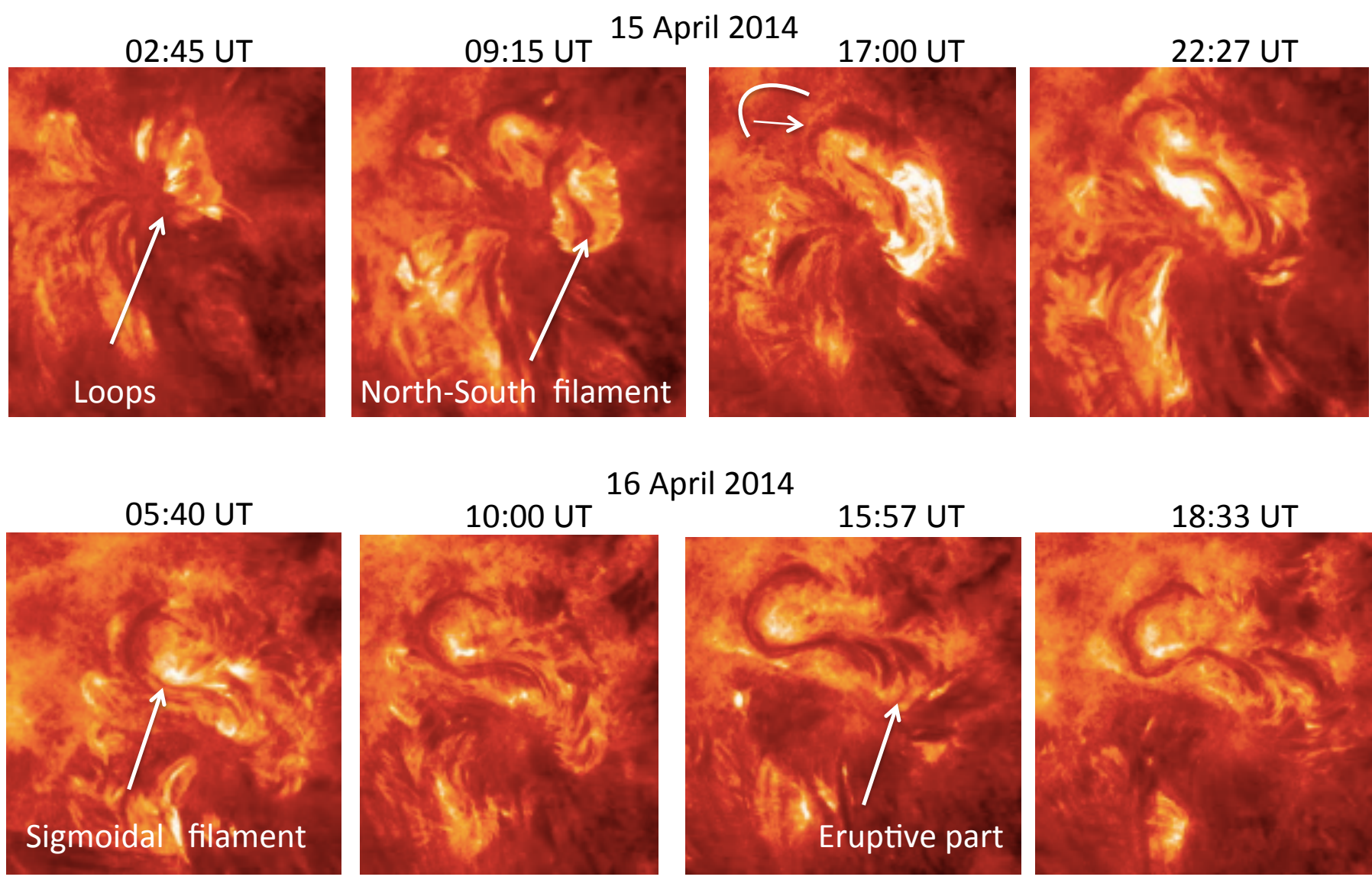

16 April 2014
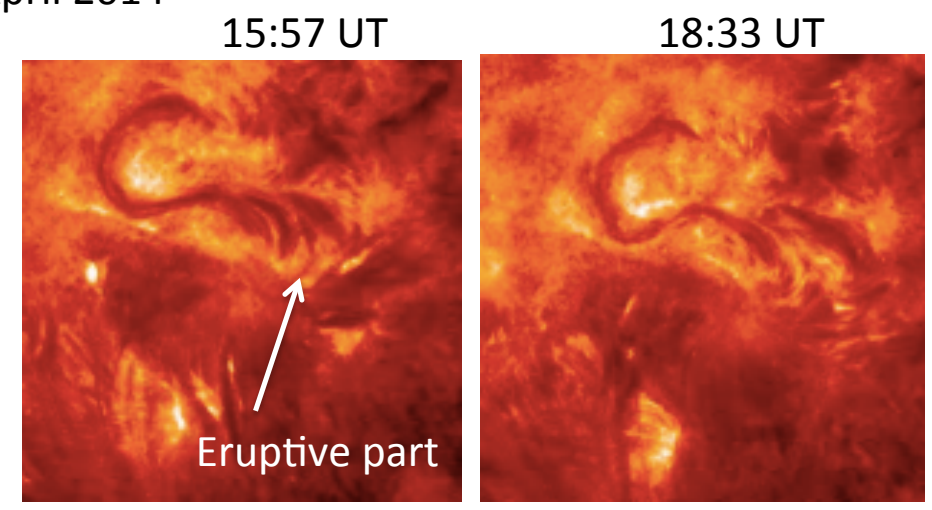

Fig. 3. Evolution of the filaments in the north part of AR 12035 between 2014 April 15 and 16 in AIA $304 \AA$ A. We note the north-south filament on April 15 at 09:15 UT and the upside-down U filament at 17:00 UT. A sigmoidal filament is formed on April 16. The FOV corresponds to the black box in Fig. 2. The temporal evolution is shown in Movie 2 (available online).

The AIA $171 \AA$ observations confirm the failed eruption (Fig. 4, bottom row). The filament is visible in absorption with a S-shaped at 10:47 UT and with a side view of the arcade overlying the western part of the filament during the eruption at 10:54 UT. The ribbons appear as bright structures along the foot points of the arcades.

\subsection{Evolution of the magnetic field}

On April 15, the active region had an overall bipolar structure characterized by a positive leading polarity $\mathrm{P} 1$ and a following negative one N1 (Fig. 5 and accompanying Movie 3, left panel). The leading polarity appeared to be constituted of a preceding compact flux distribution P1, coinciding with the umbra of the leading sunspot (Fig. 5, right panel), followed by a more disperse polarity $\mathrm{P} 2$. The positive polarity $\mathrm{P} 1$ is surrounded by a moat region with frequent bipole flux emergence and cancellation. Consequently, the positive polarity P1 is surrounded by two negative flux distributions, labeled N2 and N3 in Fig. 5, left panel. The continuum intensity (Movie 3, right panel) shows that the sunspot P1 is rotating in the clockwise direction by an angle of about 35 degrees during the two days of observations (and about 80 degrees between April 14 and 18, not shown in the movie).

Between April 15 and 16 we observe the emergence of new magnetic flux between the dispersed positive flux P2 and the following negative polarity N1 (see Movie 3). This region corresponds to the area of an arch filament system (AFS) visible in Fig. 2e. As a result of this process, part of the positive dispersed flux that constitutes the leading polarity is annihilated and the separation between the negative N1 and positive $\mathrm{P} 2$ flux distribution increases (see Movie 3). Furthermore, the leading polarity is now characterized by two compact distributions of positive flux that are well separated (see P1 and P2 in Movie 3 and Fig. 6c).

Starting from about 19:00 UT on April 15 a succession of bipoles with a larger negative polarity and a weaker positive one is seen to emerge in the north of P1 leading to an accumulation of flux in N3 (see Movie 3). Subsequently, we observe a northeast migration with a counterclockwise rotation of the newly emerged flux N3. Therefore, there is a strong shear between the clockwise rotating polarity $\mathrm{P} 1$ and the counterclockwise rotation of N3.

Contemporaneously with this migration, small concentrations of magnetic flux are seen to spread from the compact leading polarity P1 in all directions. As a result, part of the flux of $\mathrm{P} 1$ is canceled with the negative fluxes N3 and N2. The recurrent jets (visible in Movie 1) probably originate from the cancellation of $\mathrm{N} 2$ and $\mathrm{P} 1$ that may lead to magnetic reconnection producing the observed jets around the location of $\mathrm{N} 2$.

By 10:24 UT on April 16 (Movie 3 and Fig. 6c) the positive polarity of the AR is constituted of three separate (more or less compact) distributions of positive flux (see P1, P2, P3 in Fig. 6c) with a negative intrusion $\mathrm{N} 3$ at the north of the leading compact one.

The filament that is the subject of this study is located along the polarity inversion line (PIL) between the compact positive polarity P1 and the negative flux distribution N3 (arrow in Fig. 5, left panel, and blue arcades in Fig. 6). 


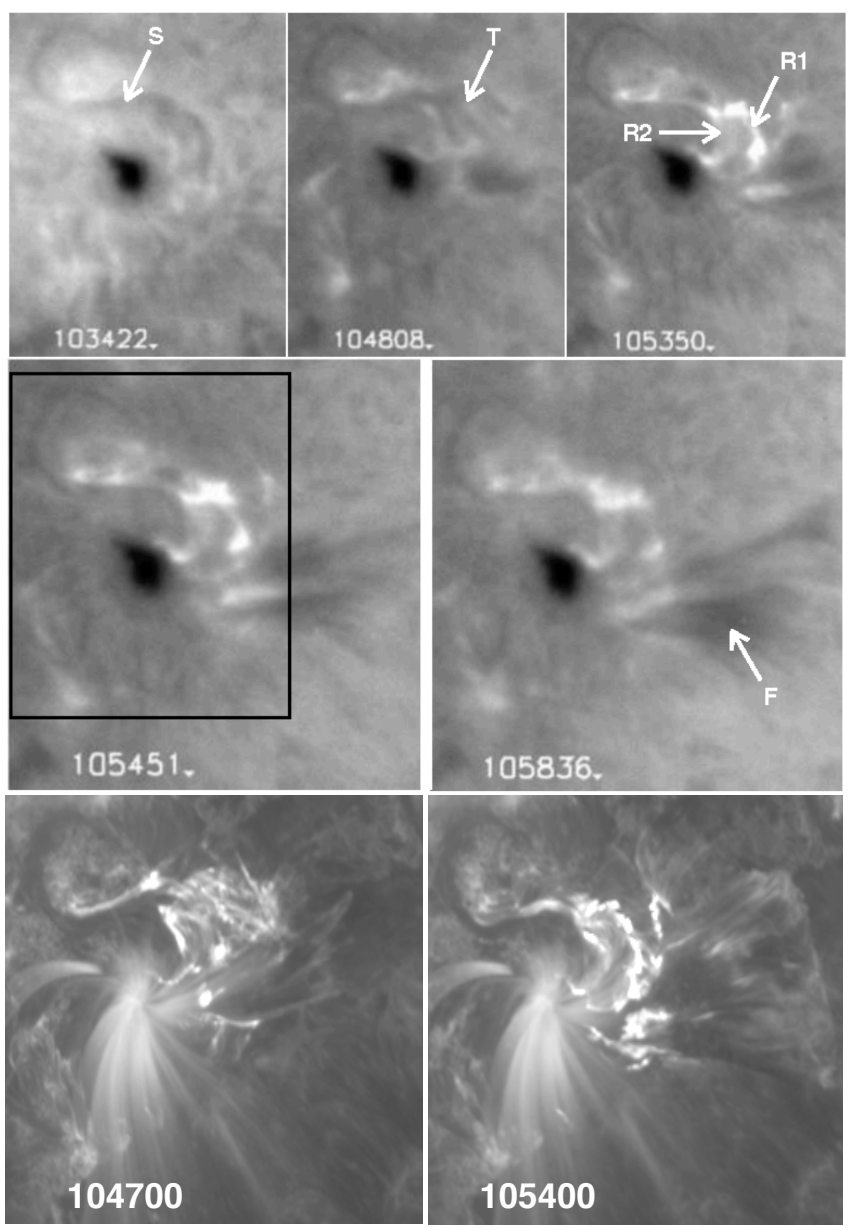

Fig. 4. $\mathrm{H} \alpha$ images of the failed filament eruption and of the flare on 2014 April 16 from 10:34-10:58 UT (from Nainital ARIES telescope, top rows). The box in the left middle image represents the FOV of the images in the top row. In the bottom row, the corresponding images in AIA $171 \AA$ at 10:47UT and 10:54 UT are shown. The FOV of the bottom panels is similar to the FOV of the middle panels. See text for more details on the S-shaped filament (S), the multiple threads (T), the two bright flare ribbons (R1, R2), and the black fan-like shapes (F).

\section{Topology of the magnetic field}

In this section we describe the key topological structures of the active region between April 15 and 16, i.e., between the time period when the nature of the flares changed from eruptive to confined.

\subsection{Potential magnetic field extrapolations}

To study the connectivity of the different flux domains and their evolution we computed a potential magnetic field model of the AR (Fig. 6, left columns). Potential configurations give robust information on the topological structures of the coronal field such as separatrices and quasi-separatrices (see Sect. 3.2 and Démoulin et al. 1996).

Since we mainly focus on the connectivity of the active region, we perform the potential extrapolation using a larger FOV provided by the HMI LOS magnetograms that includes the neighboring active regions rather then the much smaller FOV provided by the HMI SHARP data product. To this purpose the HMI LOS magnetograms of AR 12035 (and its neighboring active regions) taken at 10:24 UT on April 15 and April 16 have been remapped to the disk-center using the mapping software available through SolarSoft. As a result of this process the AR 12035 is rotated so that its center is located along the central meridian. During this process we also decreased the resolution of the images from the $\sim 0.5$ arcsec of HMI to $\sim 2$ arcsec. The subregion of the de-rotated magnetogram (containing both AR 12035 and the neighboring active regions) is then inserted at the center of a grid that is eight times larger and padded with zeros. The potential field extrapolation is performed by applying the fast Fourier transform method of Alissandrakis (1981) on this larger grid.

As Fig. 6 (left panels) shows the large-scale magnetic field is indeed bipolar, as discussed in Sect. 2.4, but the part of the active region that displays an increased level of activity is characterized by a more complex connectivity. Essentially, four flux domains are observed: the first connecting the northernmost part of the positive polarity (i.e., P0) to the negative flux N3 at its south (green field lines in Fig. 6a), the second connecting the negative polarity N3 with the leading compact positive polarity P1 (blue field lines), the third connecting this last with the negative flux $\mathrm{N} 2$ to the southeast of it (connecting field lines not shown), and the fourth is the large-scale field that connects the positive polarities $\mathrm{P} 0, \mathrm{P} 1$, and $\mathrm{P} 2$ to the following negative one (N1, orange field lines).

The anemone-like structure (blue-green field lines) is embedded in a bipolar field resulting in a breakout-like magnetic field configuration, and evolves from an northeast-southwest elongated structure on April 15 to a more circular one on April 16 (see Fig. 6, left panels).

\subsection{Quasi-separatrix layers}

Quasi-separatrix layers (QSLs, Démoulin et al. 1996) are thin layers characterized by a finite but sharp gradient in the connectivity of the magnetic field, and are defined as regions where the squashing degree $Q$ is large (Titov et al. 2002). QSLs are also locations where current layers easily develop and where (sliprunning) magnetic reconnection can occur (Aulanier et al. 2006; Janvier et al. 2013; Dudík et al. 2014); they often also coincide with the position of the flare ribbons (Savcheva et al. 2012, 2015, 2016; Zhao et al. 2014, 2016).

In this work we compute the $Q$-factor using the latest version of the topology tracing code (topotr, Démoulin et al. 1996), where the formula of Pariat \& Démoulin (2012) is implemented. To this purpose we define the plane at $z=0.4 \mathrm{Mm}$ as the seed plane from which the field lines are traced.

On April 15 an elongated fan-type QSL (Arrow Q1, Fig. 6b) surrounds the negative magnetic field distribution N3 to the northwest of the compact leading positive polarity $\mathrm{P} 1$ and embeds the portion of the PIL where the filament is located. This QSL essentially encloses and separates the anemone-like structure (blue-green field lines) from the global/large-scale field (orange field lines) of the active region. A spine-like QSL that starts from the northwest part of the fan-QSL and intrudes towards the central part of it is also observed (arrow Q2, Fig. 6b). Field lines that originate at the north of the spine-QSL connect to the northernmost positive polarity P0 (green lines), while those that originate at the south connect to the compact leading positive polarity P1 (blue lines, Figs. 7a and b). These latter are the ones that enclose the filament that is the object of this study.

On April 16 the fan-QSL (arrow Q1, Fig. 6d) displays a more circular and less elongated shape, while the spine-QSL (arrow Q2, Fig. 6d) now originates from the center of the fan-QSL 

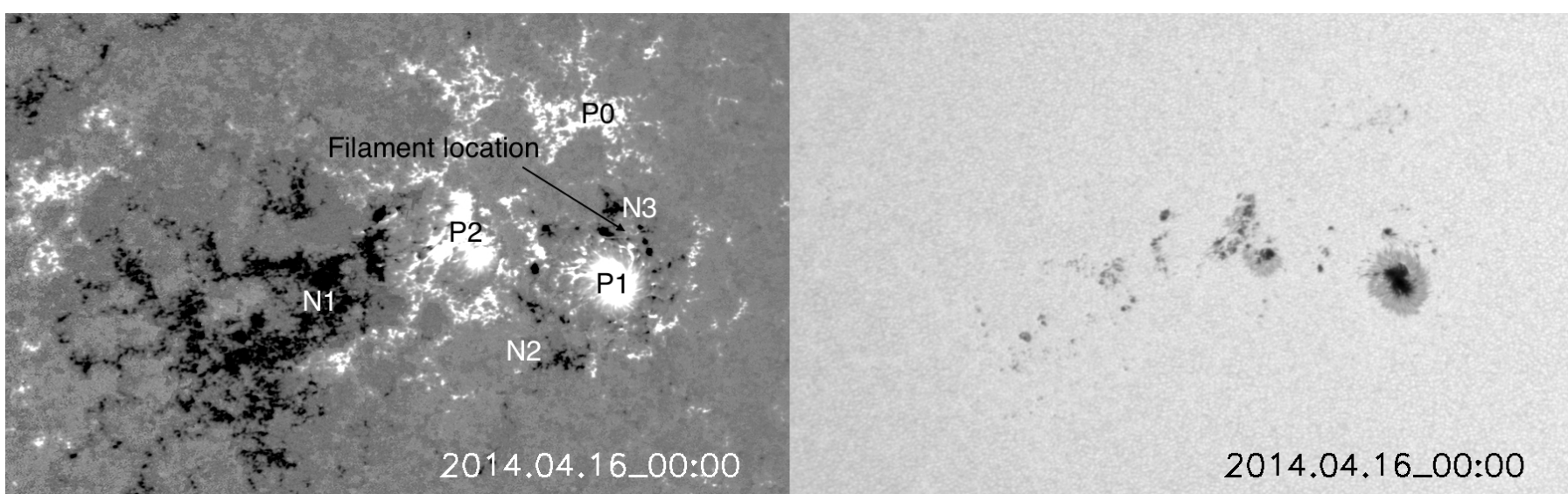

Fig. 5. HMI SHARP data projected and remapped to a cylindrical equal area (CEA) Cartesian coordinate system. Distribution of the radial component of the magnetic field (left) and of the HMI continuum (right). The FOV is $[901 \times 551]$ CEA pixels. The color scale for the magnetic field is saturated at \pm 500 Gauss and black/white indicates a negative/positive magnetic field. P0, P1, and P2 (resp. N1, N2, and N3) indicate different relevant positive (resp. negative) magnetic field distributions discussed in the text. The temporal evolution is shown in Movie 3 (available online).

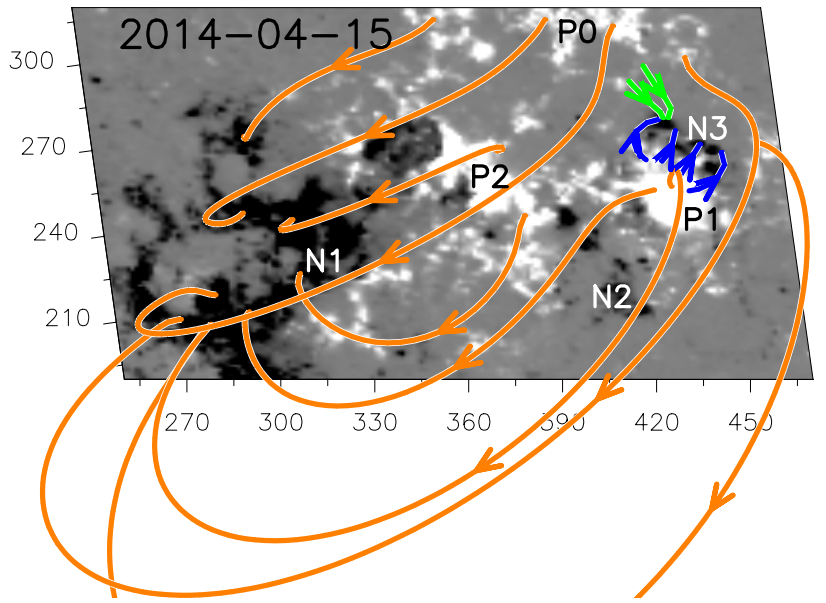

(a)

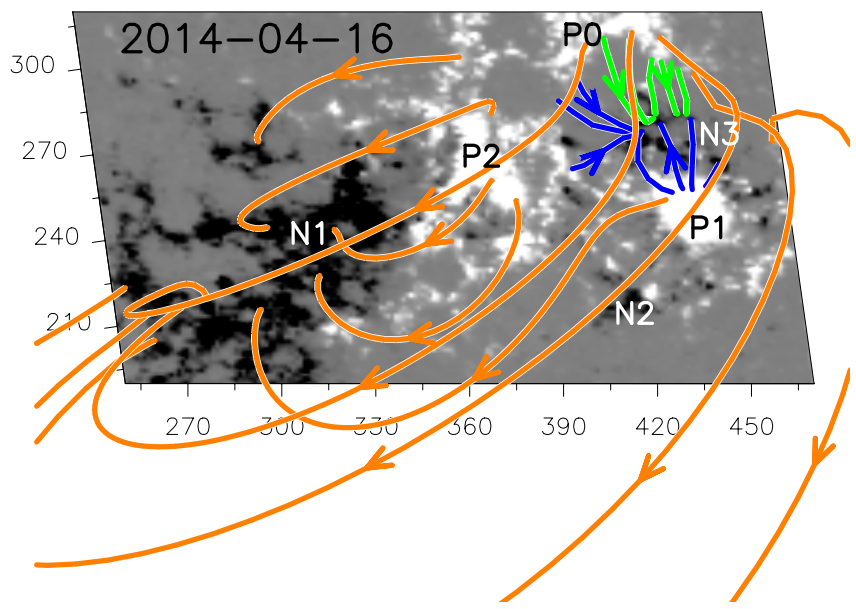

(c)

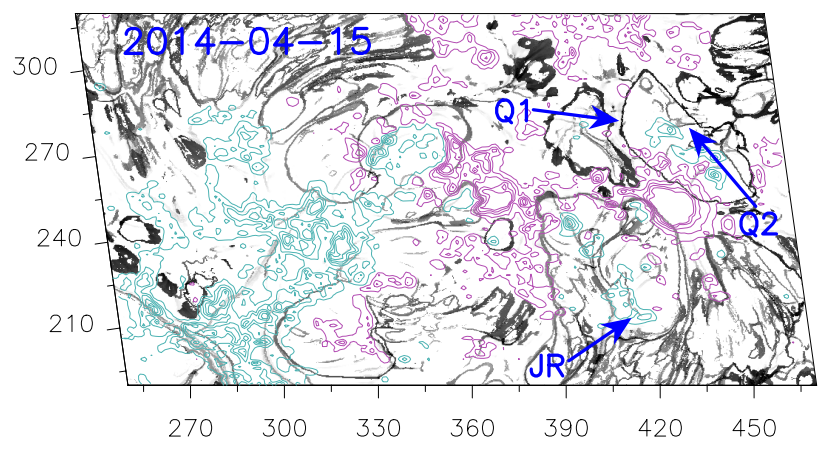

(b)

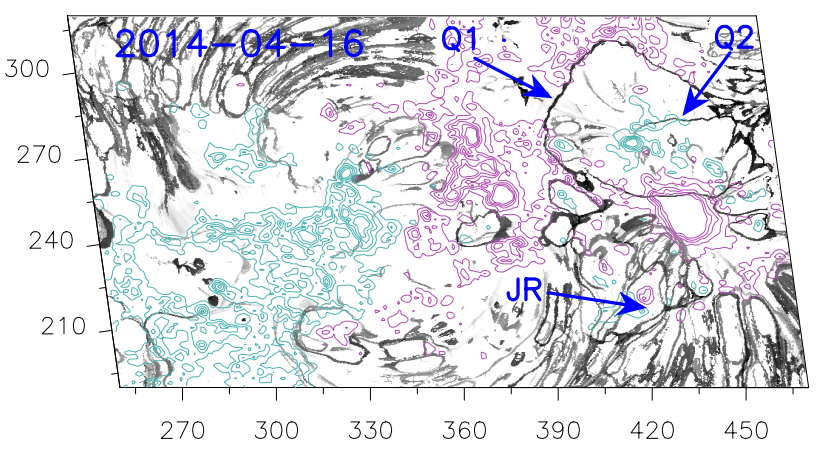

(d)

Fig. 6. Magnetic field distribution (LOS component) of AR 12035 together with some representative potential field lines (left panels) and QSL maps (right panels) for April 15 (top) and April 16 (bottom). The QSL maps (gray color scale) are computed at $z=0.4$ Mm above the photosphere (see Sect. 3.2). The color scale for the magnetic field is saturated at \pm 300 Gauss and black/white indicates a negative/positive magnetic field, while the magenta/cyan contours indicate positive/negative magnetic field values of $\pm 100, \pm 300, \pm 500, \pm 700$, and \pm 900 Gauss. The axis indicates Mm from the bottom left corner of the larger remapped HMI LOS magnetogram and is used to perform the potential field extrapolation (see Sect. 3.1 for more detail). Q1 and Q2 indicate the QSLs relevant for the eruption, while JR indicates the region where the recurrent jets occurred (see Sect. 3.2). 


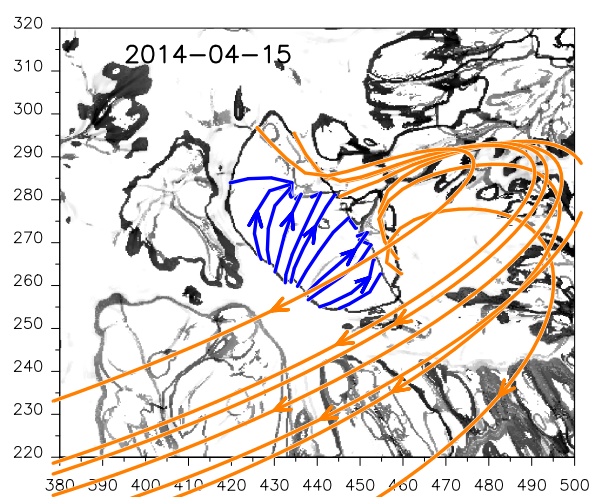

(a)

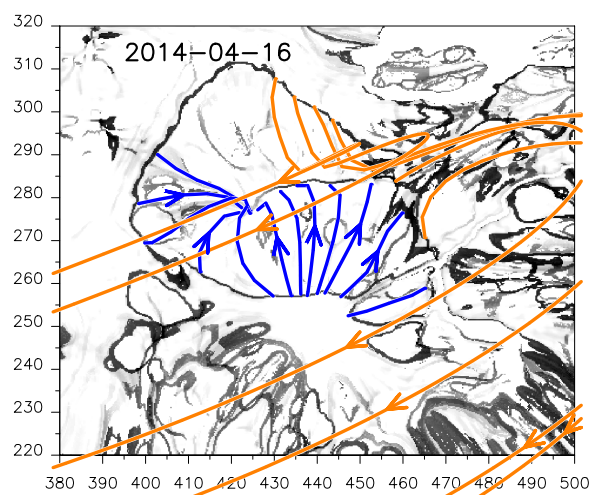

(d)

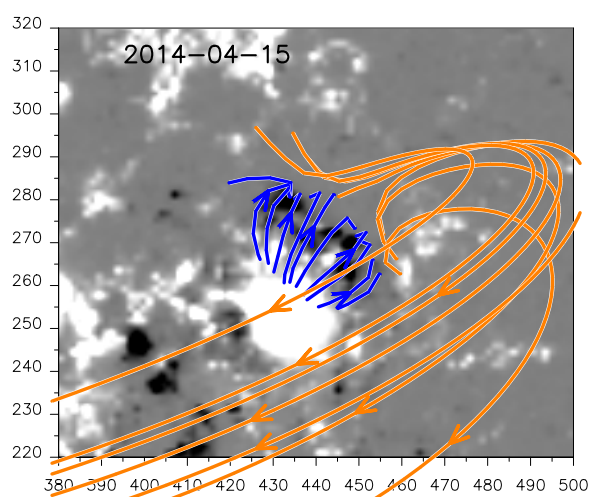

(b)

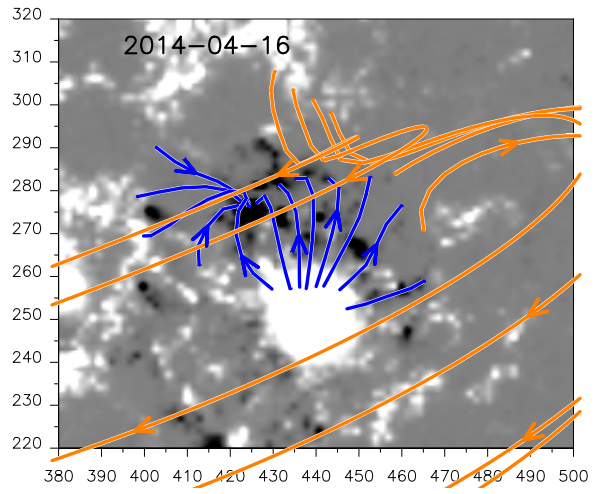

(e)

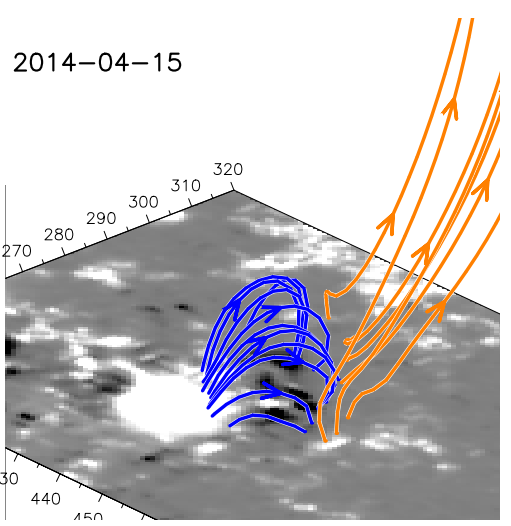

(c)

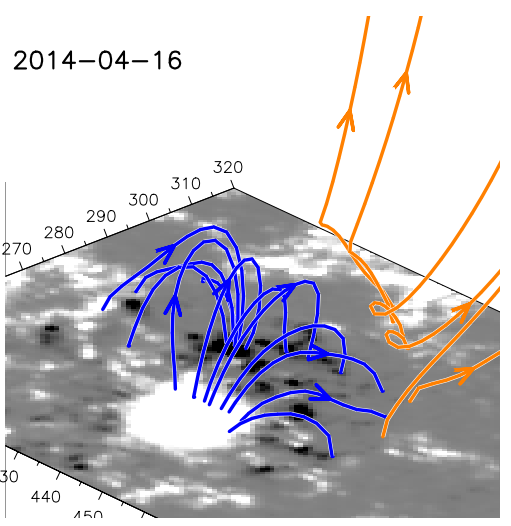

(f)

Fig. 7. Zoomed-in views of the distribution of the QSL maps at $z=0.4 \mathrm{Mm}$ (gray color scale, left) and of the photospheric (line-of-sight component) magnetic field (gray color scale, middle, right), as well as selected magnetic field lines for April 15 (top) and April 16 (bottom). For better visibility field lines in panels (c), f)) are vertically stretched by a factor of 2 . The color scale for the magnetic field is saturated at \pm 300 Gauss and black/white indicates a negative/positive magnetic field.

circle and extends westward. This magnetic field configuration indicates the presence of an elongated, locally $2 \mathrm{D}$, hyperbolic flux tube (HFT) that separates the two lobes of the anemonelike magnetic field configuration from each other and from the overlying field. This is confirmed by the vertical distribution of $Q$ along the plane passing through the spine-QSL (Fig. 8, left panels). The 2D cuts show that a local "null-point-like" configuration is achieved in both cases, but the two lobes are more symmetric on April 16 than they are on April 15.

A second, less-pronounced, more complex system of QSLs is present around the region (arrow JR, Fig. 6) where the recurrent jets are observed. However, this latter does not intersect the QSL labeled Q1, suggesting that the jet-producing region and the flaring region are not directly connected to each other (although propagating Alfvén waves may still induce a causality connection between the two parts of the active region).

While QSLs are robust topological features essentially determined by the connectivity of the magnetic field, their exact morphology depends on the actual magnetic field model used to compute them (Sun et al. 2013). To compute the $Q$-factor we used the simplest magnetic field compatible with the given boundary, i.e., the current-free magnetic field. Despite this very simple assumption we note (1) that the computed fan-QSL of the flaring region closely matches the circular flare ribbons; (2) that similarly to the computed fan-QSL, the flare ribbon actually crosses the compact leading polarity; (3) that a brightening is observed approximately at the location of the spine-QSL; and
(4) that the jet-associated brightening does not cross the flareassociated ribbons (Movie 1). This evidence suggests that the magnetic field model used is sufficient to capture the key features of the event.

As a final remark, we note that the discrepancy between the computed fan-QSL (Fig. 6) and the circular brightening ribbon (Fig. 2) is probably also due to the simplistic magnetic field model used. This can be seen in Fig. 10 of Sun et al. (2013) where the QSLs computed using both a potential field and a nonlinear force-free field (NLFFF) are compared. The fan QSL is relatively round in the potential field model, but displays a more sigmoidal shape in the NLFFF model that actually accounts for the shear present in the configuration. This is compatible with our configuration where the counterclockwise motion of the polarity N3 and the clockwise rotation of the sunspot P1 definitely introduced a degree of shear that the potential field model does not capture.

\section{Discussion}

A parameter that allows the estimation of the stability of a given magnetic field configuration is the decay index. Briefly, a magnetic flux rope embedded in an external magnetic field $\left(B_{\mathrm{ex}}\right)$ is unstable to perturbations if the axis of the flux rope has a height $z$ above the photosphere where the decay index of the external 


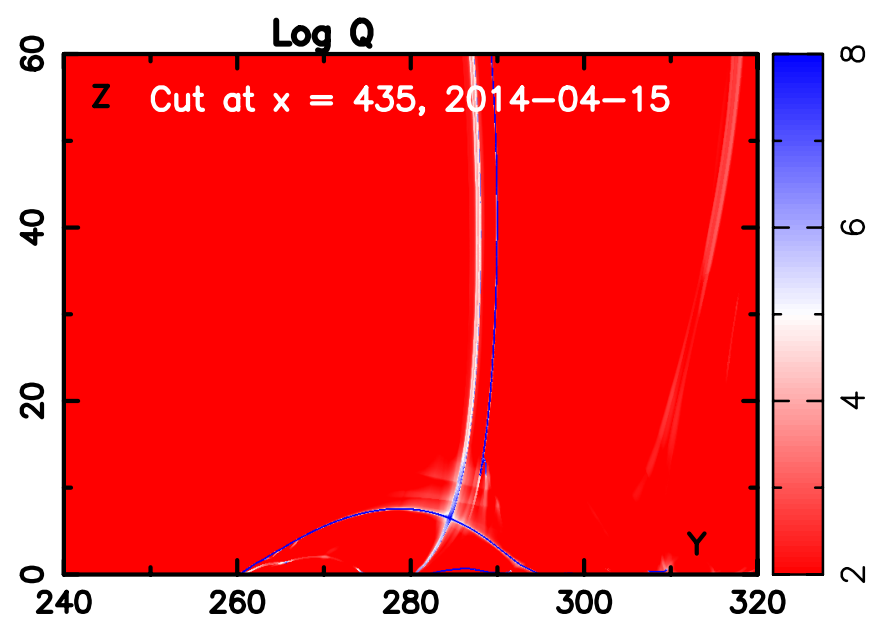

(a)

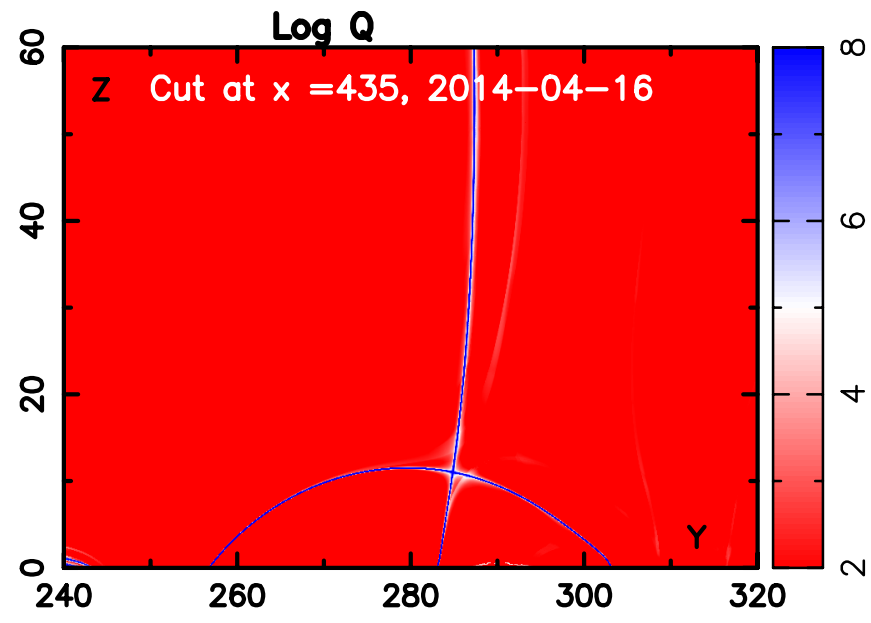

(c)

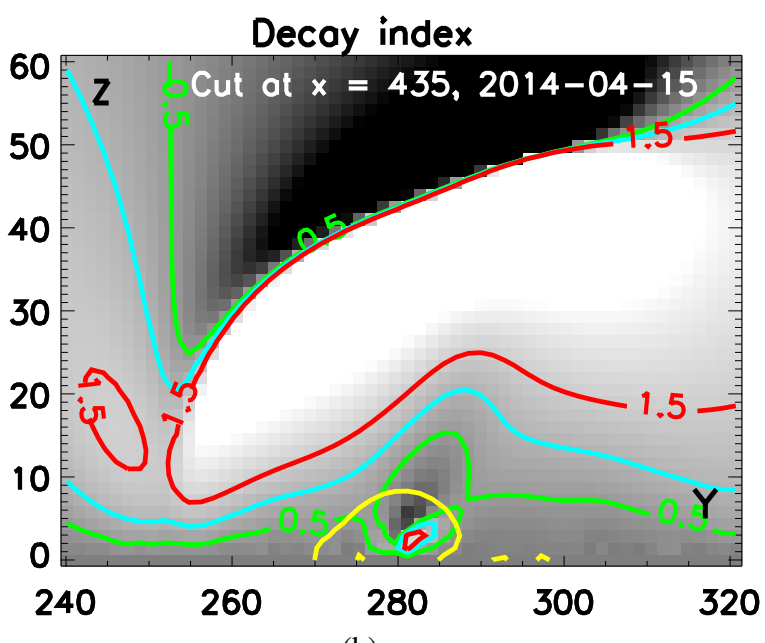

(b)

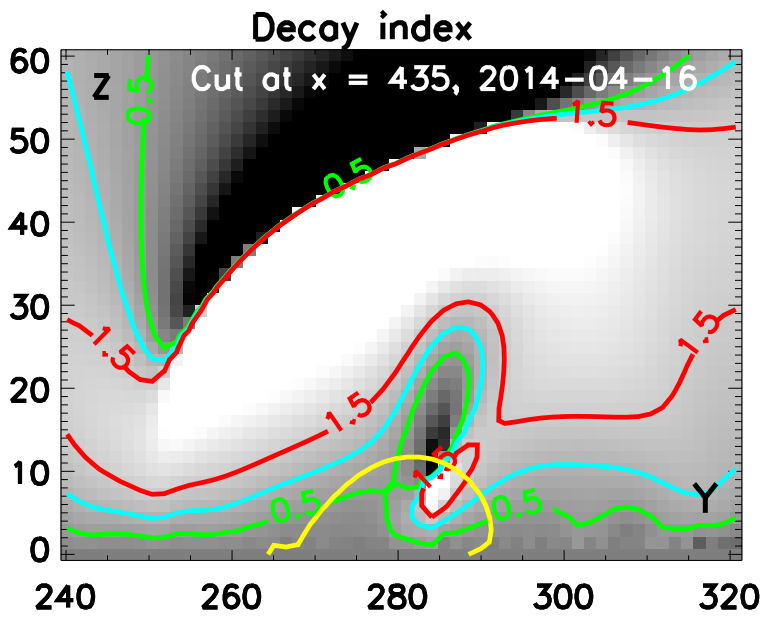

(d)

Fig. 8. Projected 2D view of the squashing degree $Q$ (left panels, see Sect. 3) and of the decay index (gray color scale, right panels) along a plane passing through $x=435$ (see Fig. 7). The HFT corresponds to the intersection of the two high $Q$ regions in the left panels. The green, cyan, and red contours on the right panels indicate isocontours of decay index $n=0.5,1$, and 1.5 , respectively. The yellow contour indicates the polarity inversion line. Axis units are in Mm from the bottom left corner of the larger remapped HMI/LOS magnetogram used to perform the potential field extrapolation (resp. from the photosphere) for the abscissa (resp. the ordinate).

magnetic field,

$n=-\frac{\mathrm{d} \ln B_{\mathrm{ex}}}{\mathrm{d} \ln z}$

is larger than a critical value $n_{\mathrm{cr}}$, which depends on the morphology of the flux rope (Démoulin \& Aulanier 2010; Zuccarello et al. 2015), and is in the range $n_{\text {cr }} \simeq 1.1-1.75$ (see Introduction).

To evaluate the stability of the magnetic field configuration we computed the decay index (using only the tangential component of the computed potential magnetic field) in the whole volume above the flaring region. A vertical cut of decay index along a plane passing through the approximate position of the HFT is shown in Fig. 8 (right panels). The first conclusion that can be drawn from the figure is that in the proximity of the HFT, the decay index changes sign becoming negative (and reaching very large negative values) as already shown by Török \& Kliem (2007) and as expected from its definition (Eq. (1)).

The second conclusion is that the large-scale stability of the magnetic field (away from the HFT) does not change significantly between April 15 and 16. This can be deduced by comparing the height at which the decay index is larger than the "nominal" $n=1.5$ critical value. The decay index for both days shows an initial increase with altitude (i.e., the system is more prone to erupt), followed by a decrease at even larger altitudes (i.e., the system is torus stable).

As previously discussed, both the circular photospheric-QSL (Q1, Fig. 6) and the fan-spine-like distribution of $Q$ (2D vertical cuts in Fig. 8) indicate the presence of a null-point topology in the corona. At the null-point the decay index (Eq. (1)) has a singularity and its validity is limited in this region. The distinction between torus instability or breakout-type reconnection as trigger mechanism for the eruption in this configuration is not at all straightforward (Kliem et al. 2014a). Furthermore, in configurations with a vertical magnetic field, such as the one considered in this paper (see Fig. 7, right columns), the verticality of the field lines itself prevents any tension-related confinement even in a uniform field where the decay index is zero. Therefore, for this complex magnetic field topology the analysis of the decay index does not provide a useful criterion for eruptivity.

For a configuration that displays a coronal null point, the breakout scenario is a valuable mechanism that triggers the 
eruption. In this scenario the eruption is triggered by the onset of magnetic reconnection, and its efficiency also depends on the mutual orientation of the reconnecting fields. Galsgaard et al. (2007) performed a series of MHD simulations of a dynamical flux emergence experiment in order to study the role of the mutual orientation between the emerging flux rope and the overlying field. The authors have shown that when the two system are (nearly) anti-parallel substantial reconnection is observed, while this is not the case when the flux systems are (nearly) parallel. More recent simulations of dynamical emergence have shown that interaction of (nearly) anti-parallel flux systems leads to flux rope-like eruptions, while this is not the case for (nearly) parallel systems (Archontis \& Török 2008; Archontis \& Hood 2012; Leake et al. 2013, 2014).

A comparison between the magnetic field extrapolations for April 15 and 16 shows that the inclination between the two flux systems (blue/orange field lines in Fig. 7) that are involved in the series of flares changed during these two days. The flux systems are more anti-parallel on April 15, than they are on April 16. This change in the mutual orientation of the field is in agreement with the evolution of the active region. As discussed in Sect. 2.4 the negative polarity $\mathrm{N} 3$ undergoes a counterclockwise rotation during the two days of observation, while the positive sunspot $\mathrm{P} 1$ is seen to rotate clockwise of about 35 degrees. This not only increases the shear of the magnetic arcade that supports the filament, as can be deduced from the formation of an S-shaped filament on April 16 (see Fig. 3), but also changes the orientation of the field systems making them less favorable to reconnect on April 16 with respect to April 15.

To discuss the magnetic configuration of the system we used a potential field model. While this model is able to identify the key topological structures of our active region (see Sect. 3), it has some limitations. First, the low-lying magnetic field, i.e., the one that supports the filament, is definitely in a non-potential state as the clear S-shaped structure of the filament suggests. As a consequence of this extra shear the actual inclination between the magnetic flux system that supports the filament (blue field lines in Fig. 7) and the overlying field (orange field lines) is probably larger, i.e., less anti-parallel, than predicted by the potential field model. Second, we note that the potential field model is an overrelaxed and already fully reconnected model. As a consequence, while it may seem that the discussion of the mutual orientation between the two flux systems applies only at high altitude, in reality the interaction occurs at a lower height. Actually, the blue loops anchored in the moving negative polarity N3 will just rise and collide with $\mathrm{P} 0-\mathrm{N} 1$ orange-type loops that initially have their orientation at low altitude, pushing them up and reconnecting to form the green loops (Fig. 6).

During the two days of observations all the eruptions (both full and failed) are initiated around the southern part of the negative polarity N3. The reason for this behavior can be understood from Fig. 7. This is the location of the coronal (quasi-)separator and a perturbation around the (quasi-)separator will initiate magnetic reconnection around its location. As a consequence, the tension of the confining arcade will be reduced, and the system evolves in the direction of the favorable magnetic pressure gradient, i.e., towards the (quasi-)null point. On April 15, the mutual orientation of the flux systems is favorable for reconnection along the full extension of the filament and we observe a series of full eruptions. On the contrary, on April 16, the mutual orientation is less favorable for the reconnection as we move farther away from the location of the null. The failed eruptions begin from the southern part of the negative polarity $\mathrm{N} 3$, which is more prone to reconnection (see Fig. 7e). However, the main part of the filament is located in a region where the mutual orientation of the flux systems is less favorable for magnetic reconnection and the full eruption is eventually inhibited.

\section{Conclusion}

The aim of this paper was to study the transition from eruptive to confined flares in active region NOAA 12035. This transition occurred between 2014 April 15 and April 16. On April 15, 4 of the 13 flares observed resulted in a CME, while none of the 13 flares recorded on April 16 resulted in a measurable CME.

During the two days of observation the filament evolved from two separated filaments on April 15 to a single S-shaped filament on April 16. Contemporaneously with this evolution we observed the presence of significant shear motions that were the results of clockwise/counterclockwise motions of the two magnetic polarities $(\mathrm{P} 1, \mathrm{~N} 3)$ where the arcade that supports the filament was anchored.

To study the topology of the active region we performed two potential field extrapolations, one on April 15 and one on April 16, and computed the QSLs. We found that a closed fanlike QSL exists around the location of the filament on both days. The presence of circular, closed fan-QSLs indicates the presence of a (quasi-)separator in the corona.

The presence of a null-point topology in the corona, the presence of shear motions that reduced the mutual inclination between the two flux systems achieving a configuration less favorable for reconnection, as well as the non-significant change in the theoretical stability (with respect to the torus instability scenario) between the two days, lead us to the conclusion that the breakout scenario seems the more probable scenario to describe the observed behavior. The discerning element between full and failed eruption behavior being determined by the mutual inclination of the flux systems involved in the process, namely the erupting flux and the overlying field.

Acknowledgements. F.P.Z. is a Fonds Wetenschappelijk Onderzoek (FWO) research fellow (Project No. 1272714N). F.P.Z. acknowledges the AXA Research Fund for contributing to this research. R.J. thanks the DST, Govt. of India, for the INSPIRE fellowship. We thank Dr. Wahab Uddin for providing the $\mathrm{H}$-alpha $\mathrm{Ob}$ servations. AIA and HMI data are courtesy of NASA/SDO and the AIA and HMI science teams. The SOHO/LASCO data used here are produced by a consortium of the Naval Research Laboratory (USA), Max-Planck-Institut für Sonnensystemforschung (Germany), Laboratoire d'Astrophysique Marseille (France), and the University of Birmingham (UK). SOHO is a project of international cooperation between ESA and NASA.

\section{References}

Alissandrakis, C. E. 1981, A\&A, 100, 197

Amari, T., Canou, A., \& Aly, J.-J. 2014, Nature, 514, 465

Antiochos, S., DeVore, C., \& Klimchuk, J. 1999, ApJ, 510, 485

Archontis, V., \& Hood, A. W. 2012, A\&A, 537, A62

Archontis, V., \& Török, T. 2008, A\&A, 492, L35

Aulanier, G. 2014, in IAU Symp. 300, eds. B. Schmieder, J.-M. Malherbe, \& S. T. Wu, 184

Aulanier, G., DeLuca, E. E., Antiochos, S. K., McMullen, R. A., \& Golub, L. 2000, ApJ, 540, 1126

Aulanier, G., Pariat, E., Démoulin, P., \& DeVore, C. R. 2006, Sol. Phys., 238, 347

Aulanier, G., Török, T., Démoulin, P., \& DeLuca, E. E. 2010, ApJ, 708, 314

Aulanier, G., Janvier, M., \& Schmieder, B. 2012, A\&A, 543, A110

Brueckner, G. E., Howard, R. A., Koomen, M. J., et al. 1995, Sol. Phys., 162, 357

Canou, A., \& Amari, T. 2010, ApJ, 715, 1566

Carmichael, H. 1964, NASA Spec. Publ., 50, 451

Chandra, R., Schmieder, B., Aulanier, G., \& Malherbe, J. M. 2009, Sol. Phys., 258,53 
Chandra, R., Chen, P. F., Fulara, A., Srivastava, A. K., \& Uddin, W. 2016, ApJ, 822,106

Chen, P. F. 2011, Liv. Rev. Sol. Phys., 8

Chen, Y., Du, G., Zhao, D., et al. 2016, ApJ, 820, L37

Dalmasse, K., Chandra, R., Schmieder, B., \& Aulanier, G. 2015, A\&A, 574, A37

Démoulin, P., \& Aulanier, G. 2010, ApJ, 718, 1388

Démoulin, P., Priest, E. R., \& Lonie, D. P. 1996, J. Geophys. Res., 101, 7631

Dudík, J., Janvier, M., Aulanier, G., et al. 2014, ApJ, 784, 144

Dudík, J., Polito, V., Janvier, M., et al. 2016, ApJ, 823, 41

Fan, Y., \& Gibson, S. E. 2007, ApJ, 668, 1232

Filippov, B. 2013, ApJ, 773, 10

Filippov, B. P., \& Den, O. G. 2001, J. Geophys. Res., 106, 25177

Filippov, B., Martsenyuk, O., Srivastava, A. K., \& Uddin, W. 2015, J. Astrophys. Astron., 36, 157

Forbes, T. 2010, Models of coronal mass ejections and flares, eds. Schrijver, C. J. \& G. L. Siscoe (Cambridge University Press), 159

Forbes, T. G., \& Isenberg, P. A. 1991, ApJ, 373, 294

Galsgaard, K., Archontis, V., Moreno-Insertis, F., \& Hood, A. W. 2007, ApJ, 666,516

Gibb, G. P. S., Mackay, D. H., Green, L. M., \& Meyer, K. A. 2014, ApJ, 782, 71

Gibson, S. 2015, in Solar Prominences, eds. J.-C. Vial, \& O. Engvold, Astrophys. Space Sci. Lib., 415, 323

Gibson, S. E., \& Fan, Y. 2006, ApJ, 637, L65

Gibson, S. E., Kucera, T. A., Rastawicki, D., et al. 2010, ApJ, 724, 1133

Green, L. M., Kliem, B., \& Wallace, A. J. 2011, A\&A, 526, A2

Guo, Y., Ding, M. D., Schmieder, B., et al. 2010a, ApJ, 725, L38

Guo, Y., Schmieder, B., Démoulin, P., et al. 2010b, ApJ, 714, 343

Harra, L. K., Schrijver, C. J., Janvier, M., et al. 2016, Sol. Phys., 291, 1761

Hirayama, T. 1974, Sol. Phys., 34, 323

Inoue, S., Hayashi, K., Magara, T., Choe, G. S., \& Park, Y. D. 2015, ApJ, 803 73

Isenberg, P. A., \& Forbes, T. G. 2007, ApJ, 670, 1453

Janvier, M., Aulanier, G., Pariat, E., \& Démoulin, P. 2013, A\&A, 555, A77

Janvier, M., Aulanier, G., \& Démoulin, P. 2015, Sol. Phys., 290, 3425

Jiang, C., Wu, S. T., Feng, X., \& Hu, Q. 2014, ApJ, 786, L16

Jing, J., Yuan, Y., Wiegelmann, T., et al. 2010, ApJ, 719, L56

Karpen, J. T., Antiochos, S. K., \& DeVore, C. R. 2012, ApJ, 760, 81

Kliem, B., \& Török, T. 2006, Phys. Rev. Lett., 96, 255002

Kliem, B., Su, Y. N., van Ballegooijen, A. A., \& DeLuca, E. E. 2013, ApJ, 779 129

Kliem, B., Lin, J., Forbes, T. G., Priest, E. R., \& Török, T. 2014a, ApJ, 789, 46

Kliem, B., Török, T., Titov, V. S., et al. 2014b, ApJ, 792, 107

Kopp, R. A., \& Pneuman, G. W. 1976, Sol. Phys., 50, 85
Leake, J. E., Linton, M. G., \& Török, T. 2013, ApJ, 778, 99

Leake, J. E., Linton, M. G., \& Antiochos, S. K. 2014, ApJ, 787, 46

Lemen, J. R., Title, A. M., Akin, D. J., et al. 2012, Sol. Phys., 275, 17

Liu, R., Gilbert, H. R., Alexander, D., \& Su, Y. 2008, ApJ, 680, 1508

Liu, R., Kliem, B., Török, T., et al. 2012, ApJ, 756, 59

Lynch, B. J., Antiochos, S. K., DeVore, C. R., Luhmann, J. G., \& Zurbuchen, T. H. 2008, ApJ, 683, 1192

Mandrini, C. H., Demoulin, P., Schmieder, B., et al. 2006, Sol. Phys., 238, 293

Masson, S., Pariat, E., Aulanier, G., \& Schrijver, C. J. 2009, ApJ, 700, 559

McCauley, P. I., Su, Y. N., Schanche, N., et al. 2015, Sol. Phys., 290, 1703

Pariat, E., \& Démoulin, P. 2012, A\&A, 541, A78

Pesnell, W. D., Thompson, B. J., \& Chamberlin, P. C. 2012, Sol. Phys., 275, 3

Rachmeler, L. A., Gibson, S. E., Dove, J. B., DeVore, C. R., \& Fan, Y. 2013, Sol. Phys., 288, 617

Romano, P., Zuccarello, F. P., Guglielmino, S. L., \& Zuccarello, F. 2014, ApJ, 794, 118

Savcheva, A. S., Green, L. M., van Ballegooijen, A. A., \& DeLuca, E. E. 2012, ApJ, 759, 105

Savcheva, A., Pariat, E., McKillop, S., et al. 2015, ApJ, 810, 96

Savcheva, A., Pariat, E., McKillop, S., et al. 2016, ApJ, 817, 43

Schmieder, B., Aulanier, G., Demoulin, P., et al. 1997, A\&A, 325, 1213

Schmieder, B., Aulanier, G., \& Vršnak, B. 2015, Sol. Phys., 290, 3457

Schou, J., Scherrer, P. H., Bush, R. I., et al. 2012, Sol. Phys., 275, 229

Sturrock, P. A. 1966, Nature, 211, 695

Sun, X., Hoeksema, J. T., Liu, Y., et al. 2013, ApJ, 778, 139

Sun, X., Bobra, M. G., Hoeksema, J. T., et al. 2015, ApJ, 804, L28

Thalmann, J. K., Su, Y., Temmer, M., \& Veronig, A. M. 2015, ApJ, 801, L23

Titov, V. S., Hornig, G., \& Démoulin, P. 2002, J. Geophys. Res., 107, 1164

Török, T., \& Kliem, B. 2005, ApJ, 630, L97

Török, T., \& Kliem, B. 2007, Astron. Nachr., 328, 743

Tripathi, D., Reeves, K. K., Gibson, S. E., Srivastava, A., \& Joshi, N. C. 2013, ApJ, 778, 142

Yashiro, S., Gopalswamy, N., Akiyama, S., Michalek, G., \& Howard, R. A. 2005, J. Geophys. Res., 110, A12S05

Zhao, J., Li, H., Pariat, E., et al. 2014, ApJ, 787, 88

Zhao, J., Gilchrist, S. A., Aulanier, G., et al. 2016, ApJ, 823, 62

Zhu, C., \& Alexander, D. 2014, Sol. Phys., 289, 279

Zuccarello, F. P., Soenen, A., Poedts, S., Zuccarello, F., \& Jacobs, C. 2008, ApJ, 689, L157

Zuccarello, F. P., Jacobs, C., Soenen, A., et al. 2009, A\&A, 507, 441

Zuccarello, F. P., Seaton, D. B., Mierla, M., et al. 2014, ApJ, 785, 88

Zuccarello, F. P., Aulanier, G., \& Gilchrist, S. A. 2015, ApJ, 814, 126

Zuccarello, F. P., Aulanier, G., \& Gilchrist, S. A. 2016, ApJ, 821, L23 